\title{
Environmental and Societal Factors Affect Food Choice and Physical Activity: Rationale, Influences, and Leverage Points
}

Sarah L. Booth, Ph.D., Vitamin K Laboratory, Jean Mayer USDA Human Nutrition Research Center on Aging at Tufts University, Boston, MA; James F. Sallis, Ph.D., F.A.C.S.M., Department of Psychology, San Diego State University, San Diego, CA; Cheryl Ritenbaugh, Ph.D., M.P.H., Kaiser Permanente Center for Health Research, Portland, OR; James O. Hill, Ph.D., Center for Human Nutrition, University of Colorado Health Sciences Center, Denver, CO; Leann L. Birch, Ph.D., Department of Human Development and Family Studies, Pennsylvania State University, University Park, PA; Lawrence D. Frank, Ph.D., College of Architecture, Georgia Institute of Technology, Atlanta, GA; Karen Glanz, Ph.D., M.P.H., Cancer Research Center of Hawaii, University of Hawaii, Honolulu, HI; David A. Himmelgreen, Ph.D., Department of Anthropology, University of South Florida, Tampa, FL; Michael Mudd, Corporate Affairs, Kraft Foods, Inc., Northfield, IL; Barry M. Popkin, Ph.D., Department of Nutrition, Carolina Population Center, University of North Carolina, Chapel Hill, NC; Karyl A. Rickard, Ph.D., R.D., C.S.P., F.A.D.A., Nutrition and Dietetics Program, School of Allied Health Sciences, Indiana University School of Medicine, Indianapolis, IN; Sachiko St. Jeor, Ph.D., R.D., Nutrition Education and Research Program, University of Nevada School of Medicine, Reno, NV; Nicholas P. Hays, M.S., Energy Metabolism Laboratory, Jean Mayer USDA Human Nutrition Research Center on Aging at Tufts University, Boston, MA.

\section{Introduction}

Dietary and physical activity behaviors that affect health are influenced by a wide variety of forces; changes in these behaviors require interventions and commitment to action at multiple levels. ${ }^{1,2}$ Education-based obesity-prevention strategies (e.g., mass-media promotion of healthy foods and promotion of healthy physical activity habits through schools) are viewed as the most useful and the most feasible to implement. ${ }^{3}$ Implicit in these strategies is the focus on the individual. ${ }^{2}$ Education-based strategies have met with limited long-term success in changing behavior, ${ }^{4}$ however, perhaps owing to a general lack of supporting environmental modifications.

There is increasing recognition of the importance of the environment in shaping behavior, yet strategies that focus on changing environmental factors are much less familiar, and may therefore require partnerships with relevant sectors outside traditional health domains. As described in greater detail by Economos et al., ${ }^{5}$ partnerships among researchers, educators, government, and industry have demonstrated success in smoking reduction at the population level. Interventions such as taxation and advertisement regulations have been instrumental in pro- moting smoking cessation in the United States and are used by agriculture and agribusiness interests to promote specific food consumption patterns. Similar models of collaborations or interventions may be successful in changing food intake and physical activity, and may potentially result in such desirable outcomes as prevention and reduction of obesity. ${ }^{6}$ It is important to appreciate the interaction among multiple environmental factors and that complex behavior changes are dependent on different influences at different levels.

In Working Group II, we took on the task of identifying broader contextual, environmental, societal, and policy variables that may improve our understanding of people's eating and physical activity behaviors and may lead to new directions for influencing shifts in behavior. Ecologic models of behavior, and most health promotion models, specify that health behaviors be influenced by biologic, demographic, psychological, social/cultural, environmental, and policy variables. However, the research base that identifies specific important environmental and policy variables is very limited., ${ }^{1,7-11}$ Nevertheless, there are several reasons that support the need to identify environmental and policy influences on physical activity and eating behaviors. 
The Need to Understand Environmental and Policy Influences

It can be argued that Americans live in an environment that does not favor a balance between physical activity and food intake. ${ }^{12}$ It is easy to find and purchase a large variety of foods. Portion sizes have grown larger, in part because consumers value a greater quantity of food for a lower price. ${ }^{13}$ This exaggerated availability of palatable and varied food is a constant challenge to people's drive to eat. Our current society is structured so that most people do not need to be physically active during a typical day. For example, land use policies not only facilitate dependence on automobiles, but also hinder alternative modes of transport that demand more physical activity (e.g., walking or cycling).${ }^{14}$ Opportunities for physical activity seem to be disappearing from daily life, whereas more enticements to be physically inactive are introduced through laborsaving devices and an expanding leisure industry whose products often require inactivity. The current environment favors an imbalance between food intake and physical activity, therefore, which can contribute to obesity and chronic diseases. ${ }^{6}$

Environment and policies may affect everyone in the population; even if such environmental influences on behavior are weak, they can influence large segments of the population on a daily basis. Foods served at schools and worksites affect everyone in those settings, and every driver on a given street sees signs for fast food outlets. All residents of a neighborhood are affected by the "walkability" of their surroundings, and international comparisons show that the United States has the least "walkable" cities and the lowest rate of walking as a means of transportation. ${ }^{15}$

Limited adoption of healthful behaviors and poor maintenance of behavioral change, frequently observed in individually based interventions, ${ }^{4}$ may be partially explained by a failure to alter environments in which it is difficult to make healthful choices. In addition, environmental supports for healthful eating and physical activity are distributed unevenly throughout the population. Understanding environmental influences may help explain socioeconomic status and cultural differences in health behaviors and health outcomes. ${ }^{16}$

Ecologic models of behavior lead to predictions that efforts to change behavior will be most effective when they are carried out on multiple levels: individual, social and cultural, environmental, and policy. ${ }^{17,18}$ Thus, educational interventions that target individuals should be most effective when society provides environments and policies that encourage people to use the knowledge and skills they have learned. Environmental and policy changes should be most effective when combined with programs that motivate and enable people to obtain healthful foods and use opportunities for physical activity.
Developing Hypotheses About Environmental, Societal, and Policy Influences on Physical Activity and Eating Behaviors

\section{Approach}

Despite hypotheses that environmental variables are strong influences on physical activity and eating behavior, few studies have investigated these influences. We determined that the next step toward achieving more healthful food intake and activity patterns was to identify specific environmental, societal, and policy variables that are hypothesized to affect physical activity and eating behaviors. A panel of experts from diverse professional fields was assembled to form a working group, consistent with similar models of partnerships that have successfully changed behaviors, as evaluated by Economos et al. ${ }^{5}$ We anticipated that there would be many environmental and policy influences, so we began a database that listed the hypothesized influences. The working group constructed the database over a series of three meetings. The technique of knowledge mapping ${ }^{19}$ was used to systematically nominate and organize a hierarchy of environmental and societal factors as they affect food and physical activity choices. This was complimented by a review of key literature, as summarized here and in Appendices 1 and 2. A similar approach was recently proposed by Swinburn et al. ${ }^{20}$ The working group used knowledge mapping to identify the agencies, institutions, or industries that have the most direct responsibility for each influence. These groups are the leverage or control points because they can be involved in change strategies. Identifying intervention leverage points may also suggest short-term and longterm research and intervention priorities.

\section{Framework}

Concurrent with the development of the database, we developed the portion of the framework that dealt with variables outside the individual to complement the portion of the framework developed by Gazdag et al. ${ }^{21}$ Three types of environmental and policy influences were conceptualized in a hierarchical fashion: specific behavior settings where people live their lives; primary or proximal leverage points that control behavior settings; and secondary or distal leverage points that have indirect, but still important influences on behavior settings.

The environmental component of the framework begins with the microenvironments or "behavior settings" in which the behavior or lifestyle takes place (Figures la and $1 \mathrm{~b}$ ). The behavior or lifestyle was defined as either an eating or physical activity pattern assumed to be related to chronic disease and obesity. Within the physical activity domain, both physical activity and physical inactivity were considered because these two types of behaviors are typically affected by different sets of environmental and household level factors. ${ }^{7}$ Because eating, physical 


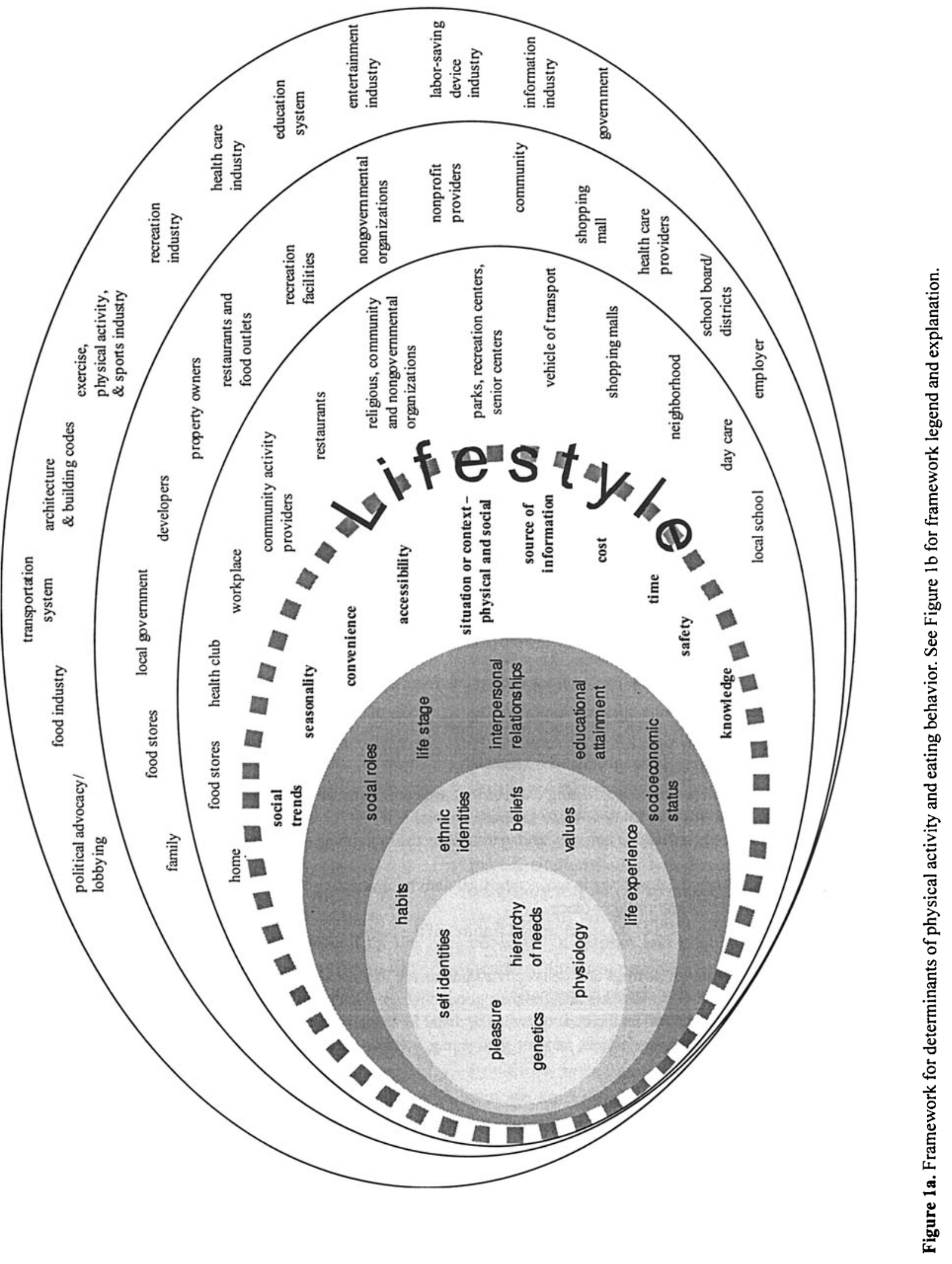




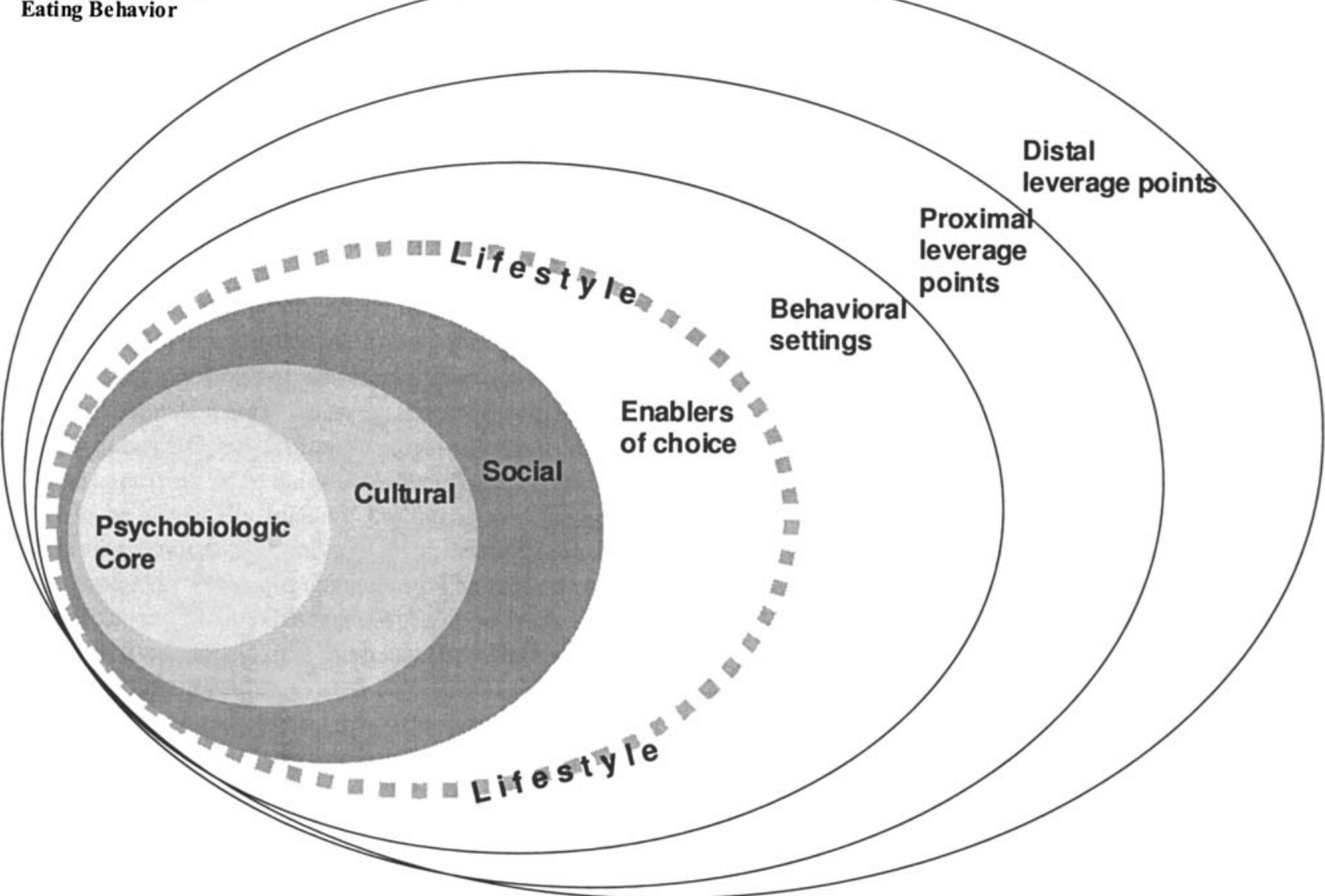

Figure 1b. Layers of the Framework.

Psychobiologic Core: Genetically programmed metabolism and behavior-instinctive behavior, innate values related to survival, which are essentially immutable. Early conditioned behaviors (e.g., positive and negative reinforcement of pleasure, pain, etc.) and experiential learning, physiologic state; these are behavioral and metabolic phenotypes expressed within a given environment. The psychobiologic core also includes current health status.

Cultural: Personal life experienced, "inherited" values and beliefs (e.g., ethnic and cultural identity), self-identity within immediate social/cultural surroundings.

Societal: Roles and relationships, "acquired" values and beliefs, how society views the individual and vice versa, i.e., self-identity within broader social/cultural environment, broader societal values (e.g., social trends). This layer interacts with the cultural layer: how society views you affects how you view yourself.

Enablers of Choice: Most proximal factors affecting choices that are commonly identified as enhancers or barriers to change. These factors tend to be the ones most focused on in order to facilitate change.

Lifestyle: Visible physical activity and eating behavior choices made by the individual, may be a mix of who they are and who they would like to be.

Behavior Settings: Physical and social settings in which physical activity and eating behaviors take place and choices are made-the situational context within which behavior takes place.

Proximal Leverage Points: Controllers of the structure and features of the microenvironment that affect the physical activity and eating behavior choices.

Distal Leverage Points: All behavior settings and macroenvironments are influenced by additional layers of factors, either directly or indirectly (e.g., controllers of the raw materials and finished goods that consumers purchase or are exposed to, along with the laws, policies, economics, politics, etc. that affect the controllers). The distal leverage points also include some multidimensional factors that pervade all levels and that shape attitudes, beliefs, and knowledge, e.g., media. 


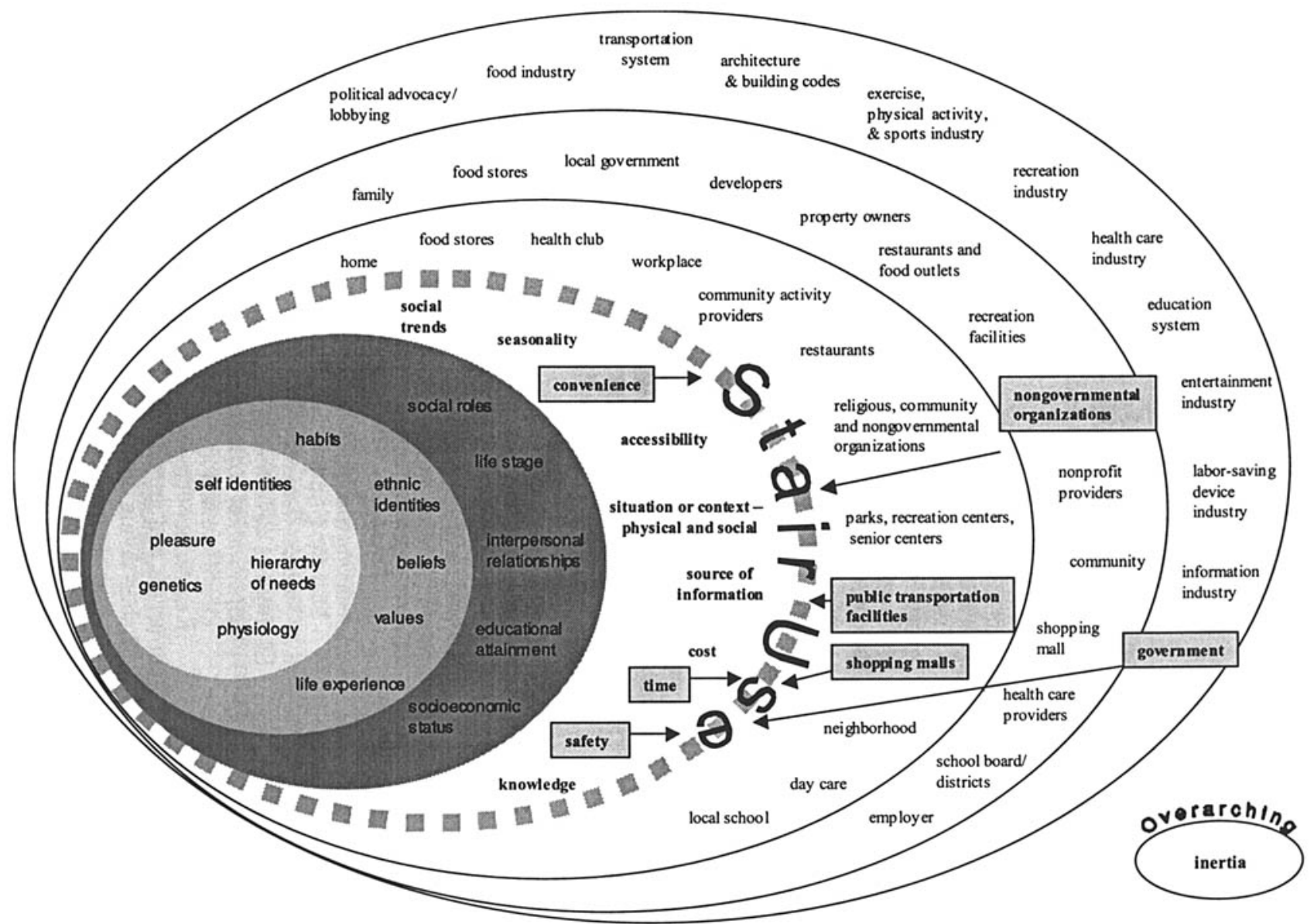

Figure 2. Framework for determinants of stair use in public transportation facilities and shopping malls. See Figure $1 \mathrm{~b}$ for framework legend and explanation.

activity, and physical inactivity can occur in many settings, 13 types of relevant behavior settings were identified. These behavior settings were then applied to the framework, but should not be considered all-inclusive. When specific intervention examples (Appendix 3) were applied to the framework to demonstrate its utility (Figure 2 ), some behavior settings were renamed and others were added.

\section{Influences}

Each behavior setting can contain many influences. For example, eating in the home may be influenced by the presence of high-calorie snacks or alternative snacks like fruits. Physical activity in the home may be influenced by the presence of exercise equipment, whereas a computer connected to the Internet may influence physical inactivity. The working group was constrained by time and therefore nominated only representative influences. Although the database is large, it is far from complete.

Each influence within a behavior setting was considered the basic unit in the database and selected characteristics of influences were recorded. When this article was written, 122 specific influences had been entered into the database (Table 1). The impact of the influence on eating or physical activity was determined by collective judgment and coded on a 3 -point scale ( 1 = low, 2 = medium, 3 $=$ high). The changeability of the influence also was coded on a 3 -point scale ( $1=$ difficult, $2=$ medium, $3=$ easy). $A$ high changeability score means that it is possible to change the influence in a healthful direction. The product of impact $X$ changeability was considered to be a useful guide regarding influences to target for short-term intervention or research. Green and Kreuter ${ }^{22}$ also advocated these concepts of impact and changeability and their role in health promotion planning.

The group rated only a minority of influences on physical activity and eating patterns to have easy changeability; most were considered difficult to change (Table 2). For those influences that were considered difficult to change, such as laborsaving devices in the home and bag lunches taken to school, the impact of successful change was predominantly rated moderate to high. This may indicate that the most meaningful environmental changes will be difficult to achieve. Although these are subjective rat- 


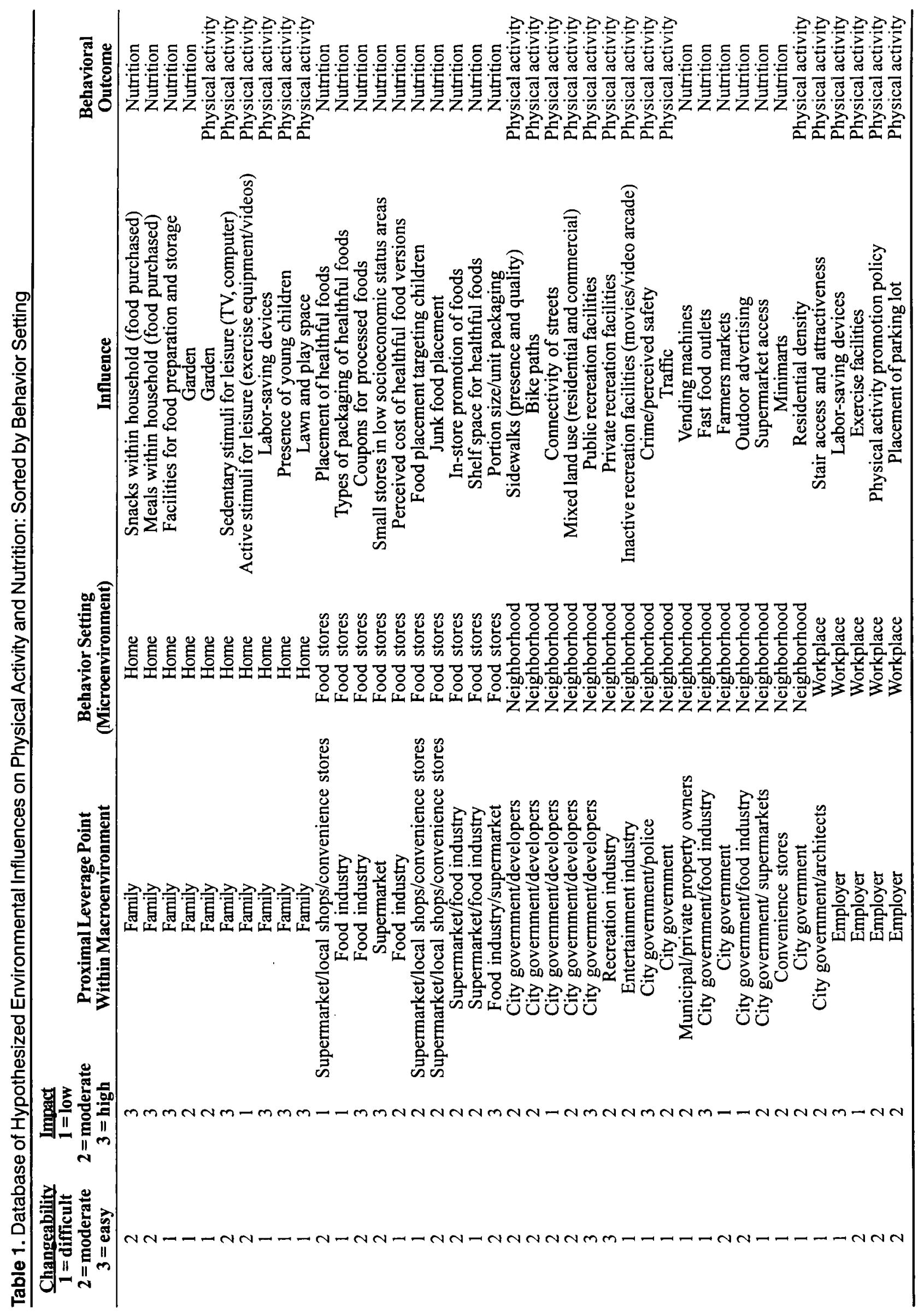




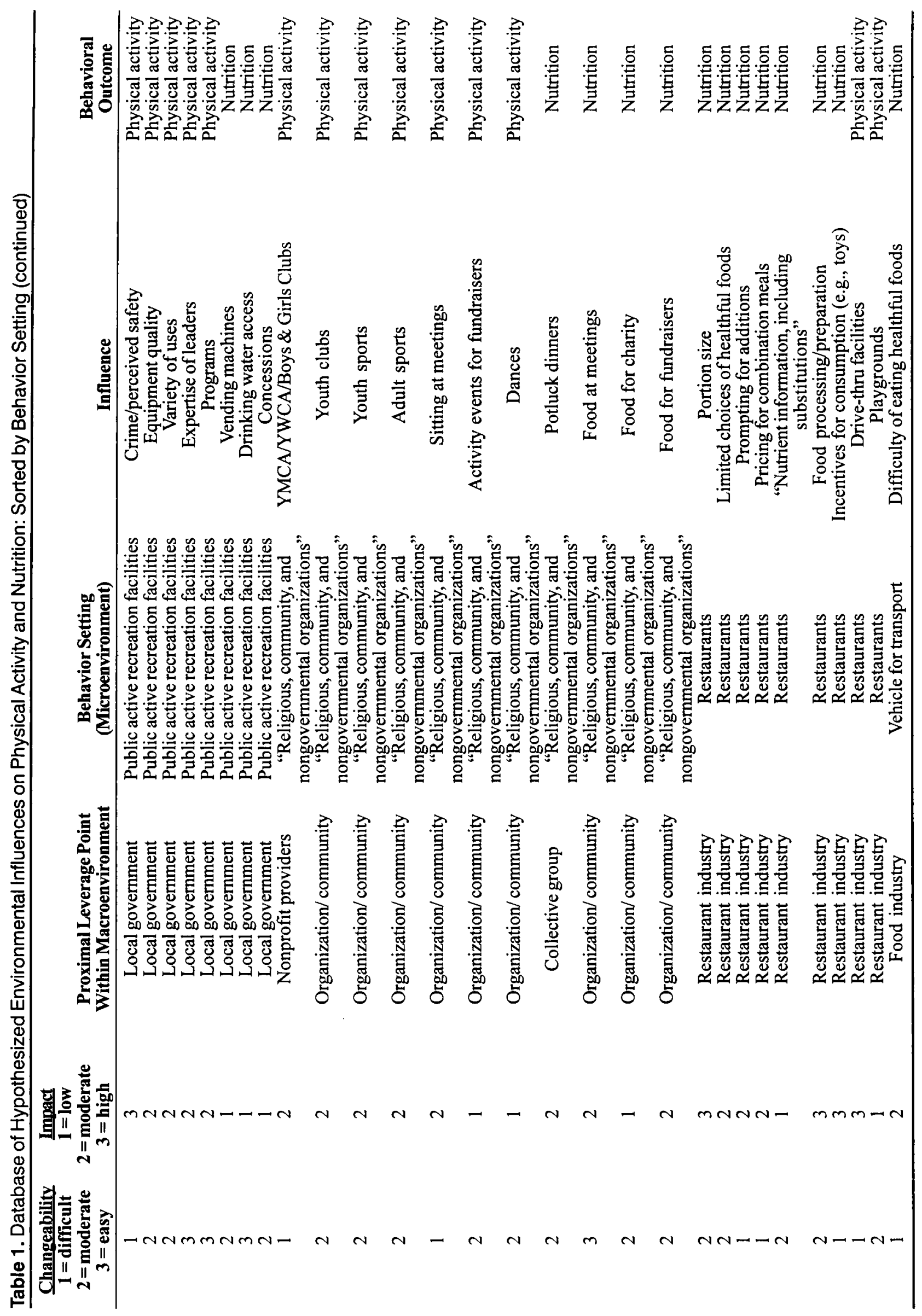




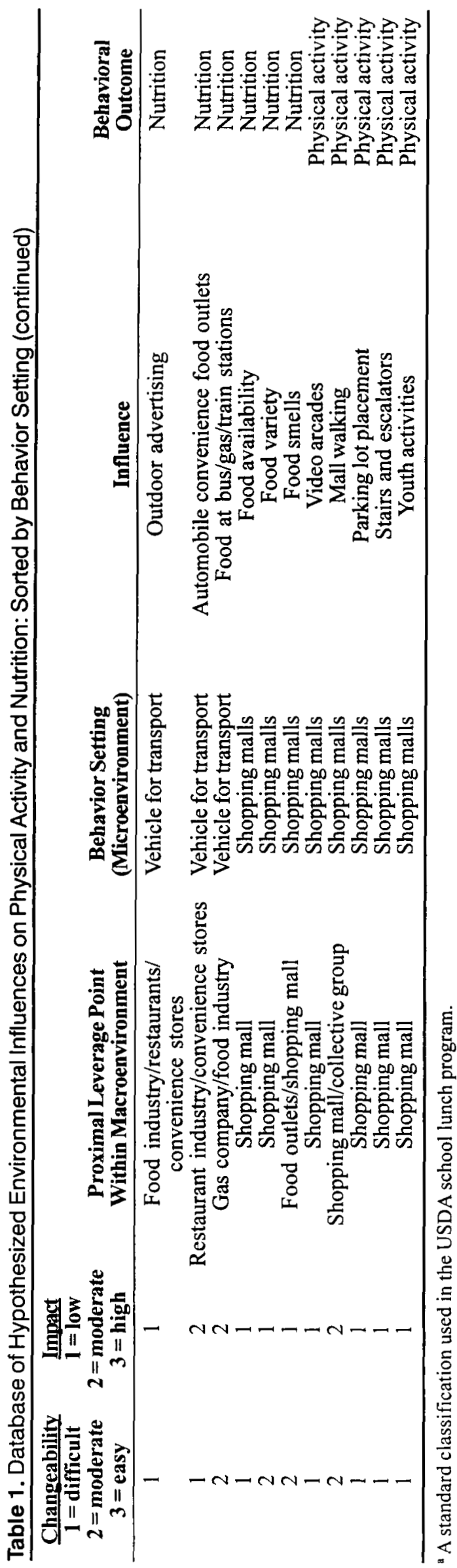

ings, the working group had the perception that influences on physical activity were no less difficult to change than eating habits, but were likely to have more impact once successful change was achieved. The lower impact ranking of influences on eating habits may be the result of bias associated with the working group members' greater collective experience in nutrition research, intervention, and policy compared with that of physical activity. As more empirical evidence becomes available, these ratings of impact and changeability can be modified accordingly.

\section{Leverage Points}

To enhance the relevance of this group's efforts to the development of interventions, the working group identified potential leverage points that may be used to change each influence. These leverage points are depicted as the outermost layers of the framework (Figure 1). The proximal leverage point was defined as the immediate controller of a given influence on physical activity or eating patterns within a behavioral setting. Distal leverage points were identified that may have indirect control over a given influence. The distinction between proximal and distal are made relative to the influence. For example, the family is the proximal leverage point for the types of snacks purchased for consumption in the home. The distal leverage points for home snack consumption include grocery stores, the food industry that produces the snacks, multiple government agencies, and advocacy groups. In our database, the grocery store is also listed as a behavioral setting, with the food industry listed as a proximal leverage point for the portion sizes available for purchase (Table 1). A leverage point, therefore, can be defined as proximal to one influence and distal for another.

Table 3 displays nine categories of distal leverage points that the group believed to have significant influence on many specific behavioral influences within multiple behavior settings. The list of leverage points is incomplete but begins the process of identifying industries, organizations, and federal, state, and local government agencies that need to be involved in efforts to improve eating and physical activity behaviors. Because the categories of distal leverage points are very broad, substantial time and effort will be required to identify specific components of the industries and institutions most relevant for influencing nutrition and physical activity. It should also be understood that government, broadly defined, was understood to be a distal leverage point for all influences. Because government influence is so pervasive, it was not always listed, and no effort was made for the current document to identify the levels or agencies of government most related to each influence.

Table 3 also illustrates that some influences on nutrition and physical activity are not easily categorized by behavior settings. Influences like advertising strategies, health news, insurance incentives, reimbursement for pre- 
Table 2. Changeability $\times$ Impact Ratings of Physical Activity and Eating Pattern Influences

\begin{tabular}{|c|c|c|}
\hline \multirow{2}{*}{ Changeability $\quad \times \quad$ Impact } & \multicolumn{2}{|c|}{ Number of Influences } \\
\hline & Physical Activity & Eating Patterns \\
\hline 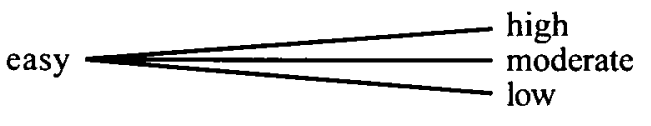 & $\begin{array}{l}2 \\
\mathbf{3} \\
2\end{array}$ & $\begin{array}{l}1 \\
2 \\
4\end{array}$ \\
\hline$\%$ of total rated easy to change & $12 \%$ & $11 \%$ \\
\hline moderate moderate $_{\text {low }}^{\text {high }}$ & $\begin{array}{l}7 \\
13 \\
9\end{array}$ & $\begin{array}{l}9 \\
11 \\
12\end{array}$ \\
\hline$\%$ of total rated moderate to change & $49 \%$ & $51 \%$ \\
\hline difficult $\underset{\text { moderate }}{\text { high }}$ low & $\begin{array}{l}10 \\
9 \\
4\end{array}$ & $\begin{array}{l}6 \\
15 \\
3\end{array}$ \\
\hline$\%$ of total rated difficult to change & $39 \%$ & $38 \%$ \\
\hline Total influences & 59 & 63 \\
\hline
\end{tabular}

ventive counseling, research policies, and professional training do not necessarily impinge on people in the places where they engage in eating, physical activity, or sedentary behaviors. These broader environmental and policy variables, nevertheless, may be important influences on behavior.

\section{Overarching Variables}

A small number of societal variables were identified as overarching across all behavioral settings and leverage points. Societal variables are seen as the context or background within which all the other variables interact. Societal variables are not likely to be changed in the short term. Overarching societal influences include competition for time, tradeoffs between multiple goals, economic rather than health motivations, and extreme demographic diversity. Societal assumptions that appear to characterize most of American culture include the higher value placed on individual rights versus the common good and the concept that "more is better." A better understanding of such societal variables may help us predict which environmental and policy interventions are most likely to be accepted or resisted by the American population. Demographic characteristics are expected to interact with specific influences such that some influences will be particularly important for various subgroups defined by age, sex, race/ethnicity, socioeconomic status, and geographic region. Because it is not possible to predict how demographics and environmental influences will interact, demographic diversity was considered an overarching societal variable that needs to be considered when planning environmental and policy interventions.

To stimulate discussion, we nominated 10 physical activity influences and 10 nutrition influences that could be considered top priorities for intervention (Table 4). The order of listing of influences reflects the number of times a working group member nominated an individual influence as a priority. Of interest was the observation that the working group members identified priorities in several behavior settings for both behaviors.

\section{Utility of the Framework and Database}

The framework and database developed by the working group are intended to contribute to a rational planning process to develop strategies to improve eating and physical activity habits in the population. The framework clearly illustrates the view of the working groups that eating and physical activity behaviors are influenced by a wide variety of internal and external factors, and all should be considered when planning interventions.

The database also suggests that eating patterns and physical activity are influenced by the complex interactions of many variables. We believe this accurately reflects the extent to which unhealthy eating and physical inactivity has become woven throughout our daily lives. We are encouraged to engage in unhealthful behaviors in multiple settings every day, and change will involve the cooperation of many corporations, government agencies, professional groups, and advocacy organizations. Because collective judgment rather than empirical research were used to create the database, the specifics can be argued. We hope such debate stimulates research rather than acrimony. The length of the list of influences and leverage points may create a sense of futility, but that is not the intention. The database helps explain why unhealthful diet and physical activity patterns are so common and resistant to long-term change. It is clear that change in the 


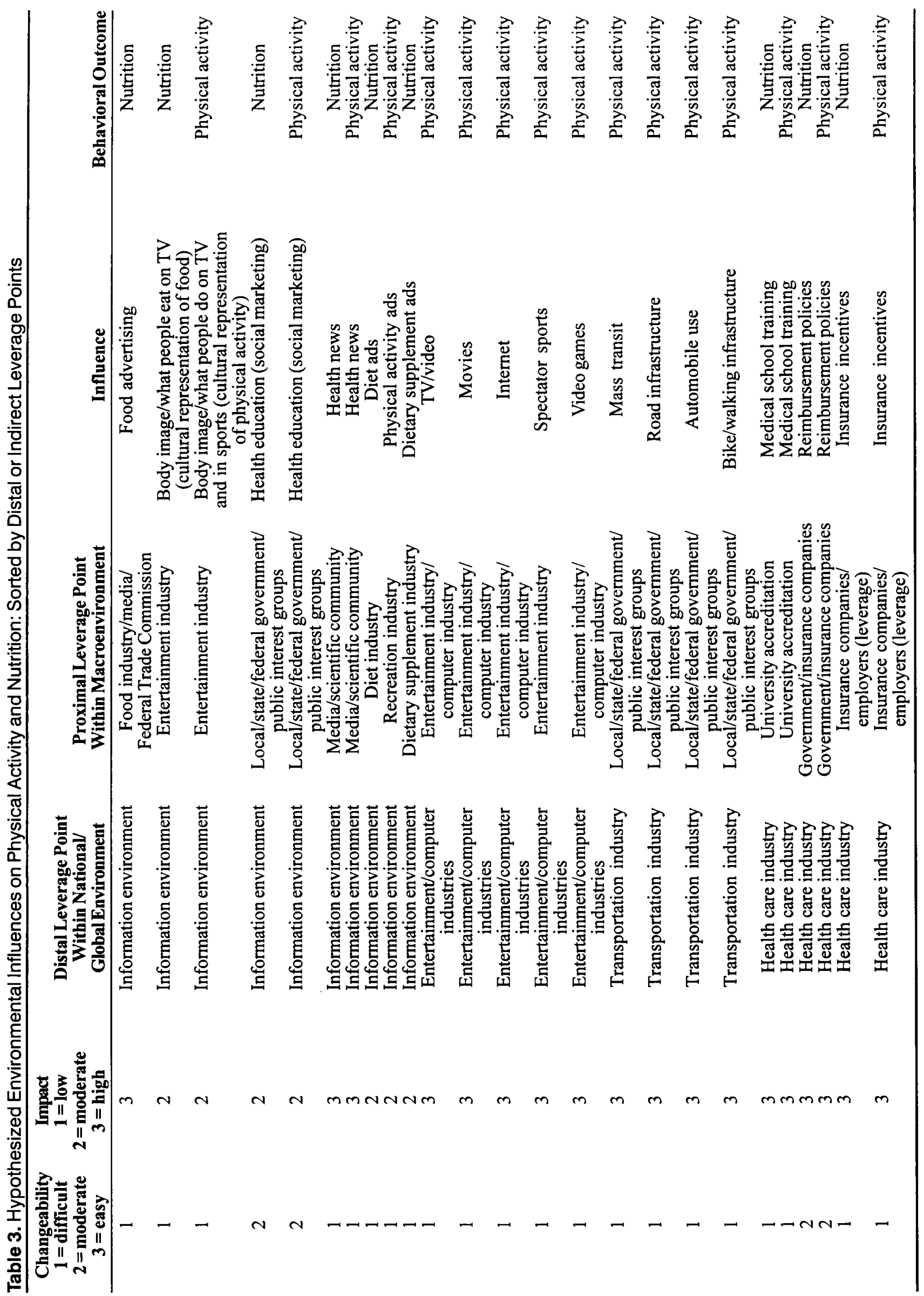




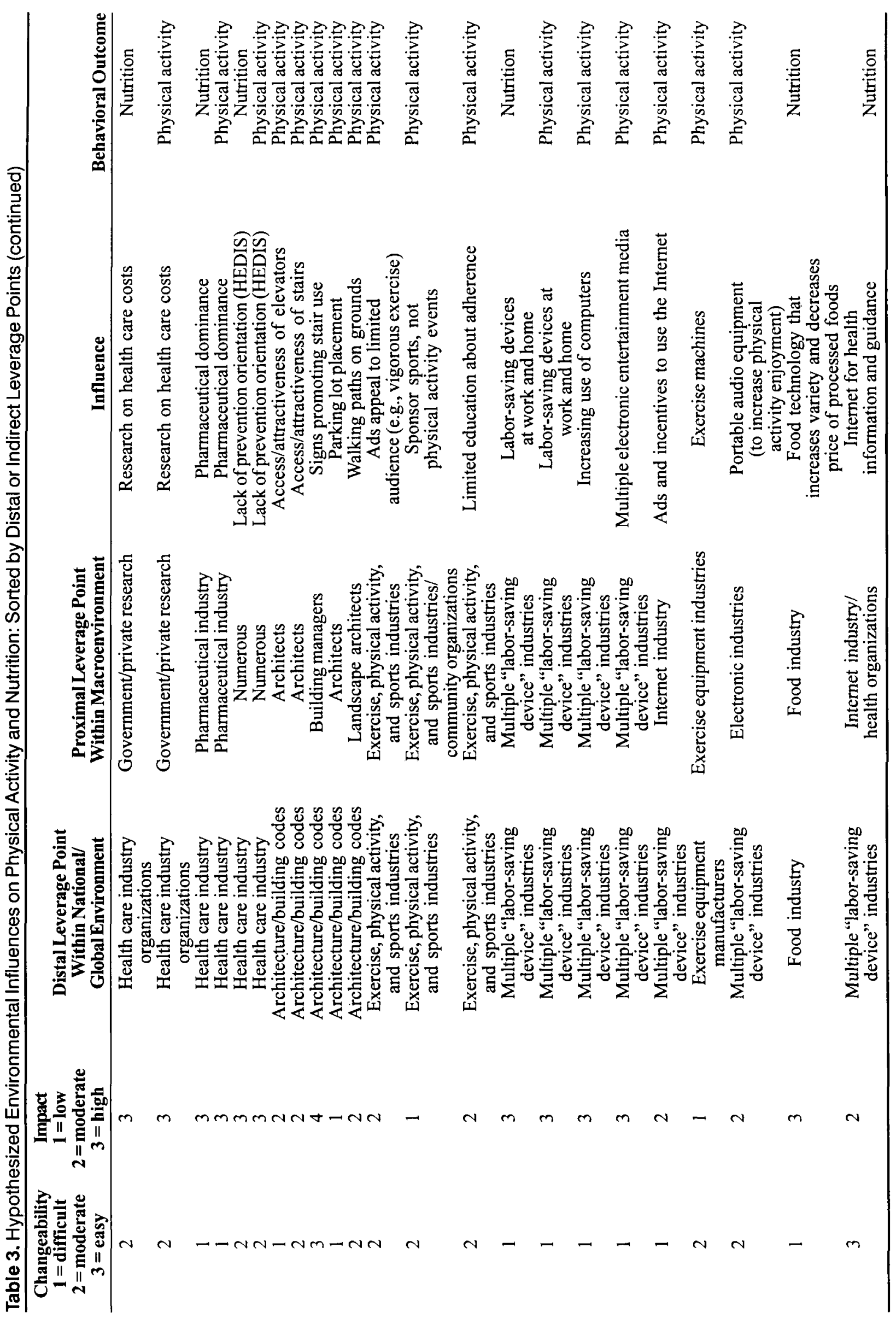




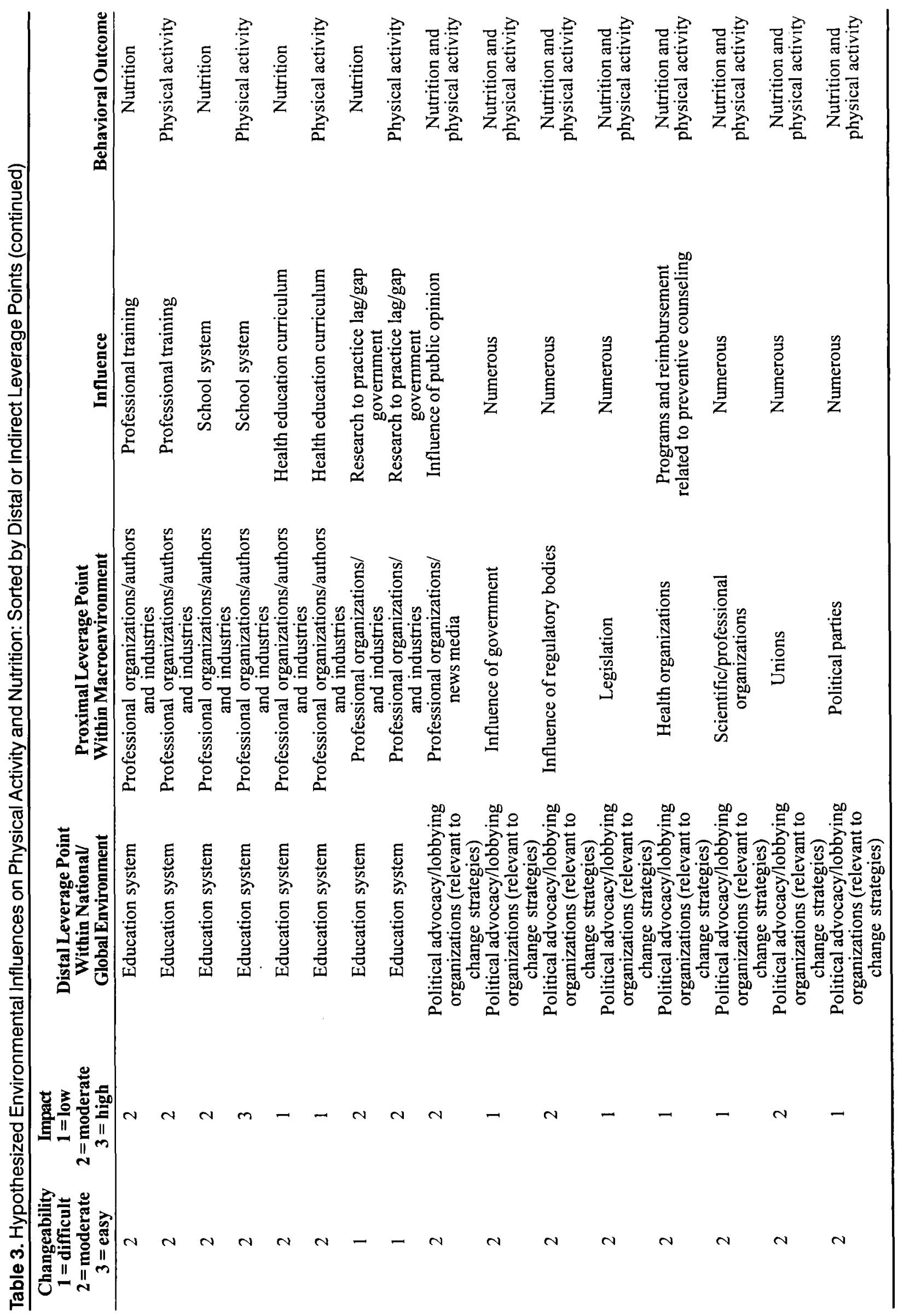


Table 4. Top Influences on Physical Activity and Eating Patterns Nominated by Working Group II

\begin{tabular}{|c|c|c|}
\hline Proximal Leverage Point & Behavioral Setting & Influence \\
\hline \multicolumn{3}{|l|}{ A. Influences on Physical Activity } \\
\hline City government and developers & Neighborhood & Public recreation facilities \\
\hline School board & School & Community use of school facilities \\
\hline Employer & Workplace & Physical activity promotion policy \\
\hline School board & School & $\begin{array}{l}\text { After school physical activity } \\
\text { programs }\end{array}$ \\
\hline School board & School & Physical education class availability \\
\hline Organizations and community & $\begin{array}{l}\text { Religious, community, and } \\
\text { nongovernment organizations }\end{array}$ & Youth sports \\
\hline City government and developers & Neighborhood & "Walkable" communities \\
\hline School board & School & $\begin{array}{l}\text { Physical education class content } \\
\text { and training }\end{array}$ \\
\hline City government and police & Neighborhood & Crime and perceived safety \\
\hline Family & Home & Sedentary stimuli for leisure \\
\hline \multicolumn{3}{|l|}{ B. Influences on Nutrition } \\
\hline Restaurant industry & Restaurants & Portion size \\
\hline Family & Home & Purchase of snacks within household \\
\hline Employer & Workplace & Cafeteria \\
\hline School board & School & Food service (self-supporting) \\
\hline School board & $\begin{array}{l}\text { School, workplace, neighborhood, } \\
\text { recreation centers }\end{array}$ & Vending machines \\
\hline Family & Home & Purchase of meals within household \\
\hline School & School & A la carte meals \\
\hline School & School & Type A meals \\
\hline City government and food industry & Neighborhood & Fast food outlets \\
\hline Food industry and supermarket & Food stores & Portion size and unit packaging \\
\hline Employer & Workplace & Company eating policy \\
\hline
\end{tabular}

A standard classification used in the USDA school lunch program.

determine how it can inform their efforts to improve the health of the population.

\section{Conclusion}

Modern society has evolved into complex environments that appear to support unhealthful patterns of eating and physical activity. These environments have developed over decades and even centuries, and we are just beginning to understand their negative effects on health. Given the large number of proposed influences, and the corresponding large number of stakeholders, it is not likely that important changes will be made quickly. It does not appear that the general public is clamoring for change in the types of environmental and policy factors discussed in this paper, and public support will be needed for extensive change. Thus, research is needed to document the extent of environmental influence and how they affect different individuals. Those findings should determine the priority placed on efforts to improve environmental supports for healthful eating and physical activity. There is also a need to tailor interventions in the face of increasing demographic diversity. In the meantime, informed hypotheses and common sense can guide practitioners to create and advocate for favorable environmental and policy changes. Consistent with ecologic models of behavior, populationwide improvements in eating and physical activity behaviors are most likely to result from interventions that change as many levels of the framework as possible, including intrapersonal, social, cultural, environmental, and policy levels.

Acknowledgements. We would like to thank Rich Schafermeyer, Procter \& Gamble Co., Cincinnati, $\mathrm{OH}$, for his valuable assistance with the knowledge mapping exercise and the development of the framework. We would also like to thank Michael Jacobson, CSPI, Washington, DC, for his contributions.

1. Glanz K, Lankenau B, Foerster S, et al. Environmental and policy approaches to cardiovascular disease prevention through nutrition: opportunities for state and local action. Health Education Quarterly 1995;22:512-28

2. King $A C$, Jeffery RW, Fridinger $F$, et al. Environmental and policy approaches to cardiovascular 
disease prevention through physical activity: issues and opportunities. Health Education Quarterly 1995;22:499-511

3. Antipatis VJ, Kumanyika SK, Jeffery RW, et al. Confidence of health professionals in public health approaches to obesity prevention. Int $\mathrm{J}$ Obes 1999;23:1004-6

4. Orleans CT. Promoting the maintenance of health behavior change: recommendations for the next generation of research and practice. Health Psychol 2000;19(suppl):76-83

5. Economos C, Brownson RC, DeAngelis MA, et al. What lessons have been learned from other attempts to guide social change? Nutr Rev 2001;59/3 part 2):\$40-\$56

6. Hill JO, Peters JC. Environmental contributions to the obesity epidemic. Science 1998;280:1371-4

7. Gordon-Larsen P, McMurray RG, Popkin BM. Determinants of adolescent physical activity and inactivity patterns. Pediatrics 2000;105:1-8

8. Guo X, Popkin BM, Mroz TA, Zhai Z. Food price policy can favorably alter macronutrient intake in China. J Nutr 1999;129:994-1001

9. Ritenbaugh C, Kumanyika SK, Jeffrey RW, et al. Caught in the causal web: a new perspective on social factors affecting obesity. Healthy Weight Journal 1999;13:88-9

10. Sallis JF, Bauman A, Pratt M. Environmental and policy interventions to promote physical activity. Am J Prev Med 1998;15:379-97

11. Teufel NI, Ritenbaugh CK. Development of a primary prevention program: insight gained in the Zuni Diabetes Prevention Program. Clin Pediatr 1999; 37:131-42

12. Poston WSC, Foreyt JP. Obesity is an environmental issue. Atherosclerosis 1999;146:201-9
13. Harnack LJ, Jeffery RW, Boutelle KN. Temporal trends in energy intake in the United States: an ecologic perspective. Am J Clin Nutr 2000;71: 1478-84

14. Frank LD. Land use and transportation interaction: implications on public health and quality of life. Journal of Planning, Education, and Research 2001, in press

15. Newman PWG, Kenworthy JR. Transport and urban form in thirty-two of the world's principal cities. Transport Reviews 1991;11:249-72

16. Messer E. Sociocultural aspects of nutrient intake and behavioral responses to nutrition. In: Galler JR, ed. Nutrition and behavior. New York, NY: Plenum Press, 1984:417-71

17. McLeroy KR, Bibeau D, Steckler A, Glanz K. An ecological perspective on health promotion programs. Health Education Quarterly 1988;15:35177

18. Stokols D. Establishing and maintaining healthy environments: toward a social ecology of health promotion. Am Psychol 1992;47:6-22

19. Novak JD, Gowin DB. Learning to learn. New York, NY: Cambridge University Press, 1984

20. Swinburn B, Egger G, Raza F. Dissecting obesogenic environments: the development and application of a framework for identifying and prioritizing environmental interventions for obesity. Prev Med 1999;29:563-70

21. Wetter AC, Goldberg JP, King AC, et al. How and why do individuals make food and physical activity choices? Nutr Rev 2001;59(3 part 2):S11-S20

22. Green LW, Kreuter MW. Health promotion planning: an educational and environmental approach, 2nd ed. Mountain View, CA: Mayfield, 1991

\section{Appendix 1}

\section{Additional Resources}

1. Position of the American Dietetic Association: dietary guidance for healthy children aged 2 to 11 years. J Am Diet Assoc 1999;99:93-101

2. American Dietetic Association. 1997 Nutrition Trends Survey. Chicago, IL: American Dietetic Association, 1997

3. Bradbard S, Michaels EF, Fleming K, Campbell P. Understanding the food choices of low-income families. Summary of findings. Alexandria, VA: U.S. Department of Agriculture, Food and Consumer Service, Office of Analysis and Evaluation, 1997

4. Bronner YL. Nutritional status outcomes for children: ethnic, cultural, and environmental contexts. J Am Diet Assoc 1996;96:891-900

5. Center for Media and Public Affairs. Food for thought III: reporting of diet, nutrition and food safety. Executive summary 1999 vs. 1997 vs. $1995-$ draft. Washington, DC: International Food Information Council Foundation, 1999

6. Center for Media and Public Affairs. Food for thought II: reporting of diet, nutrition and food safety. Executive Summary. Part I: 1997 vs. 1995. Part II: 1997. Washington, DC: International Food Information Council Foundation, 1998

7. Cheadle A, Psaty BM, Curry S, et al. Communitylevel comparisons between the grocery store environment and individual dietary practices. Prev Med 1991;20:250-61

8. Egger G, Swinburn B. An “ecological” approach to the obesity pandemic. BMJ 1997;315:477-80

9. Food Marketing Institute. Shopping for health 1997: balancing convenience, nutrition and taste. Wash- 